\title{
High Rate Golden Space-Time Trellis Coded Modulation
}

\author{
Yi Hong \\ Institute for Telecom. Research \\ University of South Australia \\ Mawson Lakes, SA 5095 \\ yi.hong@unisa.edu. au
}

\author{
Emanuele Viterbo \\ Dipartimento di Elettronica \\ Politecnico di Torino \\ Torino, Italy \\ viterbo@polito.it
}

\author{
Jean-Claude Belfiore \\ Department of Commun. Electron. \\ ENST \\ Paris, France \\ belfioredenst.fr
}

\begin{abstract}
We present a robust multidimensional trellis coded modulation scheme for a high rate $2 \times 2$ multiple-input multipleoutput (MIMO) system over both slow and fast block fading channels. Set partitioning of the Golden code [2] is designed specifically to increase the minimum determinant. The branches of the outer trellis code are labeled with these partitions. The Viterbi algorithm is applied for trellis decoding and branch metrics are computed using a sphere decoder. The general framework for code optimization is given. Performance of the proposed scheme is evaluated by simulation and it is shown that it achieves significant performance gains over uncoded Golden code on both slow and fast block fading channels. ${ }^{1}$
\end{abstract}

\section{INTRODUCTION}

Wireless networks for multimedia traffic demand high spectral efficiency coding schemes with low packet delay. Algebraic space-time codes for multiple-input multiple-output (MIMO) systems provide some very good tools so solve this challenging design problem. Wireless channels are commonly modeled as block fading. We will consider both slow and fast block fading channels, i.e., the channel coefficients are fixed over the duration of a frame or over the duration of a short block within the frame, respectively. We will show that for such channels the careful concatenation of a space-time block code with an outer trellis code provides a robust solution for high rate transmission over both slow and fast block fading channel.

Space-time trellis codes (STTCs) using PSK and QAM modulations were designed according to both rank and determinant criteria [1]. A more refined concatenated scheme enables to split these two design criteria. As an inner code, we can use a simple space-time block coding scheme, which guarantees full diversity for any spectral efficiency (e.g. Alamouti scheme). An outer code is then used to improve the coding gain.

In this paper, we consider a concatenated scheme, where the inner code is the Golden code [2] and the outer code is a trellis code. We can view this as a multidimensional trellis coded modulation (TCM), where the Golden code acts as a signal set to be partitioned. This Golden Space-Time Trellis

\footnotetext{
${ }^{1}$ This work was supported by the STREP project No. IST-026905 (MAS COT) within the Sixth Framework Programme of the European Commission, Australian Research Council (ARC) Project DP0663567, and ARC Communication Research Work (ACoRN) RN 0459498.
}

Coded Modulation (GST-TCM) scheme is appropriate for high data rate systems thanks to the great flexibility in the choice of the modulation spectral efficiency.

A first attempt to design such a scheme was made in [3]. However, the resulting ad hoc scheme suffered from a high trellis complexity. Here, we develop a systematic design approach for GST-TCM over slow and fast block fading channels. In [4-7], lattice set partitioning combined with a trellis code is used to increase the minimum square Euclidean distance between codewords. Here, it is used to increase the minimum determinant. The Viterbi algorithm is used for trellis decoding, where the branch metrics are computed using a sphere decoder for the inner code.

We propose a design approach that is based on Ungerboeck's design rules for TCM, [5,8]. We design different GST-TCM and optimize their performance according to the design criterion. It is shown that a 16 state TCM, with the spectral efficiency of 6 bits per channel use (bpcu), achieves a significant performance gain of $4.2 \mathrm{~dB}$ and $4.5 \mathrm{~dB}$ over the uncoded Golden code in slow and fast block fading channels, at an frame error rate (FER) of $10^{-3}$.

The following notations are used: $T$ denotes transpose and $\dagger$ denotes Hermitian transpose. Let $\mathbb{Z}, \mathbb{Q}, \mathbb{C}$ and $\mathbb{Z}[i]$ denote the ring of rational integers, the field of rational numbers, the field of complex numbers, and the ring of Gaussian integers, where $i^{2}=-1$. Let $\mathbb{Q}(\theta)$ denote an algebraic number field generated by the primitive element $\theta$. Let $G F(2)=\{0,1\}$ denote the Galois field of degree two. The $m \times m$ dimensional identity matrix is denoted by $\mathbf{I}_{m}$. The $m \times n$ dimensional zero matrix is denoted by $\mathbf{0}_{m \times n}$.

\section{SYSTEM MODEL}

We first consider a $2 \times 2$ MIMO system $\left(n_{T}=2\right.$ transmit and $n_{R}=2$ receive antennas) over slow block fading channels. The received signal matrix $\mathbf{Y} \in \mathbb{C}^{2 \times 2 L}$ ( $2 L$ is the frame length), is given by

$$
\mathbf{Y}=\mathbf{H X}+\mathbf{Z},
$$

where $\mathbf{Z} \in \mathbb{C}^{2 \times 2 L}$ is the complex white Gaussian noise matrix with i.i.d. samples $\sim \mathcal{N}_{\mathbb{C}}\left(0, N_{0}\right)$.

For a slow block fading channel, the channel matrix $\mathbf{H} \in$ $\mathbb{C}^{2 \times 2}$ is constant during a frame $\mathbf{X}$ and varies independently 
from one frame to another. The elements of $\mathbf{H}$ are assumed to be i.i.d. circularly symmetric Gaussian random variables $\sim$ $\mathcal{N}_{\mathbb{C}}(0,1)$. The channel is assumed to be known at the receiver.

In (1), $\mathbf{X}=\left[X_{1}, \ldots, X_{t}, \ldots, X_{L}\right] \in \mathbb{C}^{2 \times 2 L}$ is the transmitted signal matrix, where $X_{t} \in \mathbb{C}^{2 \times 2}$. If we consider fast block fading we can write

$$
Y_{t}=\mathbf{H}_{t} X_{t}+Z_{t} \quad t=1, \ldots, L
$$

where the channel matrix $\mathbf{H}_{t} \in \mathbb{C}^{2 \times 2}$ can vary every two channel uses (one block).

There are three different options for selecting inner codewords $X_{t}, t=1, \ldots, L$ :

1) $X_{t}$ are independently selected from the Golden code $\mathcal{G}$, i.e.,

$$
X_{t}=\frac{1}{\sqrt{5}}\left[\begin{array}{cc}
\alpha\left(a_{t}+b_{t} \theta\right) & \alpha\left(c_{t}+d_{t} \theta\right) \\
i \bar{\alpha}\left(c_{t}+d_{t} \bar{\theta}\right) & \bar{\alpha}\left(a_{t}+b_{t} \bar{\theta}\right)
\end{array}\right]
$$

where $a_{t}, b_{t}, c_{t}, d_{t} \in \mathbb{Z}[i]$ are the information symbols, $\theta=1-\bar{\theta}=\frac{1+\sqrt{5}}{2}, \alpha=1+i-i \theta, \bar{\alpha}=1+i(1-\bar{\theta})$, and the factor $1 / \sqrt{5}$ is used to normalize energy [2].

2) $X_{t}$ are independently selected from a linear subcode of the Golden code;

3) A trellis code is used as the outer code encoding across the symbols $X_{t}$ selected from partitions of $\mathcal{G}$.

We denote Case 1 as the uncoded Golden code, Case 2 as the Golden subcode, and Case 3 as the Golden Space-Time Trellis Coded Modulation.

In this paper, we use $Q$-QAM constellations, where $Q=2^{\eta}$ as information symbols in (3). We assume the constellation is scaled to match $\mathbb{Z}[i]+(1+i) / 2$, i.e., the minimum Euclidean distance is set to 1 and it is centered at the origin. The average energy $E_{s}$ is $0.5,1.5$ and 2.5 for $Q=4,8,16$. Signal to noise ratio is defined as $\mathrm{SNR}=n_{T} E_{b} / N_{0}$, where $E_{b}=E_{s} / q$ is the energy per bit and $q$ denotes the number of information bits per symbol. We have $N_{0}=2 \sigma^{2}$, where $\sigma^{2}$ is the noise variance per real dimension, which can be adjusted as $\sigma^{2}=$ $\left(n_{T} E_{b} / 2\right) 10^{(-\mathrm{SNR} / 10)}$.

Assuming that a codeword $\mathbf{X}$ is transmitted, the maximumlikelihood receiver might decide erroneously in favor of another codeword $\hat{\mathbf{X}}$. Let $r$ denote the rank of the codeword difference matrix $\mathbf{X}-\hat{\mathbf{X}}$. Since the Golden code is full rank, $r=n_{T}=2$. Let $\lambda_{j}, j=1, \ldots, r$, be the eigenvalues of the codeword distance matrix $\mathbf{A}=(\mathbf{X}-\hat{\mathbf{X}})(\mathbf{X}-\hat{\mathbf{X}})^{\dagger}$.

In the case of slow block fading, let $\Delta=\prod \lambda_{j}$ be the determinant of the codeword distance matrix $\mathbf{A}$ and $\Delta_{\min }$ be the corresponding minimum determinant, $\Delta_{\min }=\min \operatorname{det}(\mathbf{A})$. We call $n_{T} n_{R}$ the diversity gain and $\left(\Delta_{\min }\right)^{1 / n_{T}}$ the coding gain [1]. In the case of linear codes we can simply consider the all-zero codeword matrix and we have $\Delta_{\min } \geq$ $\min _{\mathbf{X} \neq \mathbf{0}_{2 \times 2 L}} \operatorname{det}\left(\mathbf{X X}^{\dagger}\right)$, where equality holds for infinite codes used to carve the finite constellations [2].

In order to compare two coding schemes in a $2 \times 2 \mathrm{MIMO}$ system, supporting the same information bit rate, but with different minimum determinants $\left(\Delta_{\min , 1}\right.$ and $\left.\Delta_{\min , 2}\right)$ and different constellation energies $\left(E_{s, 1}\right.$ and $\left.E_{s, 2}\right)$, we define the asymptotic coding gain as

$$
\gamma_{a s}=\frac{\sqrt{\Delta_{\min , 1}} / E_{s, 1}}{\sqrt{\Delta_{\min , 2}} / E_{s, 2}} .
$$

In the case of $L=1$, the codeword matrix $\mathbf{X}=X_{1} \in \mathcal{G}$ is a square matrix. The Golden code $\mathcal{G}$ has full rate, full rank $r=2$, and the minimum determinant is $\delta_{\min }=\frac{1}{5}$ [2]; thus $\Delta_{\min }=\delta_{\min }$ for the uncoded Golden code system.

In the case of GST-TCM over slow block fading channels

$$
\operatorname{det}\left(\mathbf{X X}^{\dagger}\right)=\operatorname{det}\left(\sum_{t=1}^{L}\left(X_{t} X_{t}^{\dagger}\right)\right) .
$$

A code design criterion attempting to maximize $\Delta_{\min }$ is hard to exploit, due to the non-additive nature of the determinant metric in (5). Since $X_{t} X_{t}^{\dagger}$ are positive definite matrices, we use the following determinant inequality [10]

$$
\Delta_{\min } \geq \min _{\mathbf{x} \neq \mathbf{0}_{2 \times 2 L}} \sum_{t=1}^{L} \operatorname{det}\left(X_{t} X_{t}^{\dagger}\right)=\Delta_{\min }^{\prime} .
$$

The lower bound $\Delta_{\min }^{\prime}$ will be adopted to design our scheme. In particular we will design trellis codes that attempt to maximize $\Delta_{\min }^{\prime}$, by using set partitioning to increase the minimum number $L^{\prime}$ of non zero terms $\operatorname{det}\left(X_{t} X_{t}^{\dagger}\right)$ in (6).

In the case of GST-TCM over fast block fading channels, the coding gain is related to

$$
\Delta_{\min }=\min _{\mathbf{X} \neq \mathbf{0}_{2 \times 2 L}} \prod_{\operatorname{det}\left(X_{t} X_{t}^{\dagger}\right) \neq 0}^{\left(L^{\prime}\right)} \operatorname{det}\left(X_{t} X_{t}^{\dagger}\right)
$$

where $L^{\prime}$ is the minimum number of non zero "det" terms.

In order to maximize this $\Delta_{\min }$ over fast block fading channels, we follow the same strategy as for slow block fading cases. Hence, the code design based on $\Delta_{\min }^{\prime}$ is robust to different channel conditions ranging from slow to fast fading.

\section{TRELLIS CODED MODULATION}

The uncoded Golden code (Case 1) and Golden subcode (Case 2) are discussed in $[3,11]$. Here, we propose a systematic design approach for Case 3 . We analyze the design problem of this scheme by using Ungerboeck style set partitioning rules for coset codes [5-7]. The design criterion for the trellis code is developed in order to maximize $\Delta_{\min }^{\prime}$, since this results in the maximum lower bound on the asymptotic coding gain of the GST-TCM over the uncoded Golden code

$$
\gamma_{a s} \geq \frac{\sqrt{\Delta_{\min }^{\prime}} / E_{s, 1}}{\sqrt{\delta_{\min }} / E_{s, 2}} .
$$

Before we design the coding scheme, we briefly recall the set partition chain in [3].

The Golden subcodes - Let us consider a subcode $\mathcal{G}_{k} \subseteq \mathcal{G}$ for $k=1, \ldots, 4$, obtained by

$$
\mathcal{G}_{k}=\left\{X B^{k}, X \in \mathcal{G}\right\}
$$




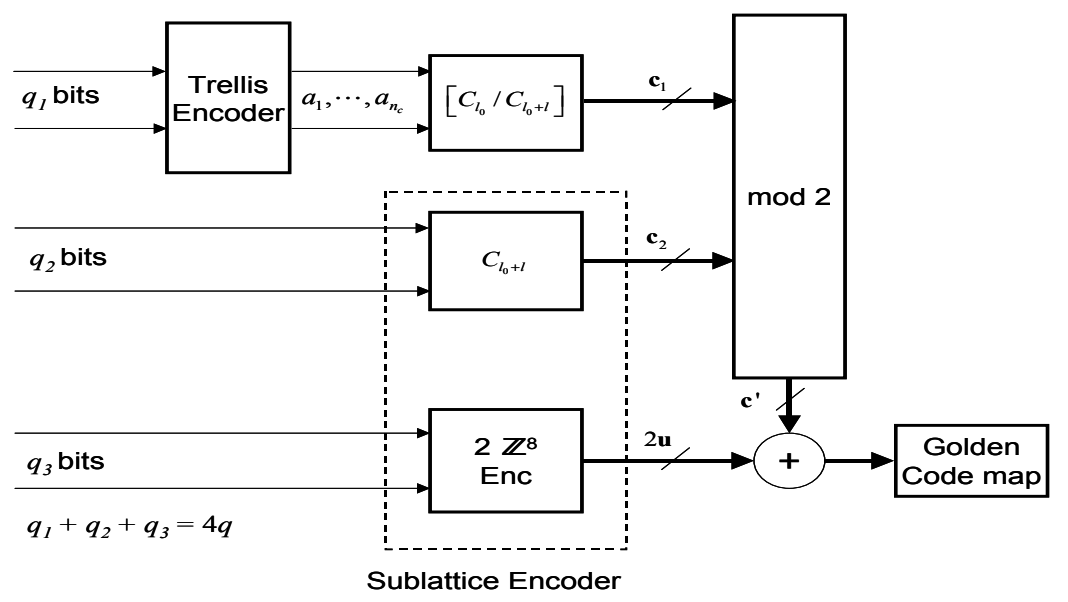

Fig. 1. General encoder structure of the concatenated scheme.

where

$$
B=\left[\begin{array}{cc}
i(1-\theta) & 1-\theta \\
i \theta & i \theta
\end{array}\right] .
$$

This provides the minimum square determinant $2^{k} \delta_{\min }$ (see Table I). It is shown that the codewords of $\mathcal{G}_{k}$, when vectorized, correspond to different sublattices of $\mathbb{Z}^{8}$. It can be verified that these lattices form the lattice partition chain

$$
\mathbb{Z}^{8} \supset D_{4}^{2} \supset E_{8} \supset L_{8} \supset 2 \mathbb{Z}^{8}
$$

where $D_{4}^{2}$ is the direct sum of two four-dimensional Shäfli lattices, $E_{8}$ is the Gosset lattice and $L_{8}$ is a lattice of index 64 in $\mathbb{Z}^{8}$. Any two consecutive lattices $\Lambda_{k} \supset \Lambda_{k+1}$ in this chain form a four way partition, i.e., the quotient group $\Lambda_{k} / \Lambda_{k+1}$ has order 4 . Let $\left[\Lambda_{k} / \Lambda_{k+1}\right]$ denote the set of coset leaders of the quotient group $\Lambda_{k} / \Lambda_{k+1}$. The lattices in the partition chain can be obtained by Construction A [9], using the nested sequence of linear binary codes $C_{k}$ listed in Table I.

Let $G_{k}$ denote the generator matrix of the code $C_{k}$ for $k=1,2,3$. We have

$$
\begin{aligned}
G_{1} & =\left[\begin{array}{llllllll}
1 & 0 & 0 & 1 & 0 & 0 & 0 & 0 \\
0 & 1 & 0 & 1 & 0 & 0 & 0 & 0 \\
0 & 0 & 1 & 1 & 0 & 0 & 0 & 0 \\
0 & 0 & 0 & 0 & 1 & 0 & 0 & 1 \\
0 & 0 & 0 & 0 & 0 & 1 & 0 & 1 \\
0 & 0 & 0 & 0 & 0 & 0 & 1 & 1
\end{array}\right] \\
G_{2} & =\left[\begin{array}{llllllll}
0 & 1 & 0 & 1 & 0 & 1 & 0 & 1 \\
0 & 0 & 1 & 1 & 0 & 0 & 1 & 1 \\
0 & 0 & 0 & 0 & 1 & 1 & 1 & 1 \\
1 & 1 & 1 & 1 & 1 & 1 & 1 & 1
\end{array}\right] \\
G_{3} & =\left[\begin{array}{llllllll}
0 & 0 & 0 & 0 & 1 & 1 & 1 & 1 \\
1 & 1 & 1 & 1 & 1 & 1 & 1 & 1
\end{array}\right]
\end{aligned}
$$

Following the track of [5-7], we consider a partition tree of the Golden code of depth $\ell$. From a nested subcode sequence $\mathcal{G} \supseteq \mathcal{G}_{\ell_{0}} \supset \mathcal{G}_{\ell_{0}+1} \supset \cdots \supset \mathcal{G}_{\ell_{0}+\ell}$, we have the corresponding lattice partition chain $\mathbb{Z}^{8} \supseteq \Lambda_{\ell_{0}} \supset \Lambda_{\ell_{0}+1} \supset \cdots \supset \Lambda_{\ell_{0}+\ell}$ where

$$
\begin{aligned}
\Lambda_{\ell_{0}} & =\Lambda_{\ell_{0}+1}+\left[\Lambda_{\ell_{0}} / \Lambda_{\ell_{0}+1}\right]=\cdots \\
& =\Lambda_{\ell_{0}+\ell}+\left[\Lambda_{\ell_{0}} / \Lambda_{\ell_{0}+1}\right]+\cdots+\left[\Lambda_{\ell_{0}+\ell-1} / \Lambda_{\ell_{0}+\ell}\right] \\
& =\Lambda_{\ell_{0}+\ell}+\left[C_{\ell_{0}} / C_{\ell_{0}+1}\right]+\cdots+\left[C_{\ell_{0}+\ell-1} / C_{\ell_{0}+\ell}\right]
\end{aligned}
$$

\begin{tabular}{|c|c|c|c|c|}
\hline Level & Subcode & Lattice & Binary code & $\Delta_{\text {min }}$ \\
\hline \hline 0 & $\mathcal{G}$ & $\mathbb{Z}^{8}$ & $C_{0}=(8,8,1)$ & $\delta_{\text {min }}$ \\
1 & $\mathcal{G}_{1}$ & $D_{4}^{2}$ & $C_{1}=(8,6,2)$ & $2 \delta_{\text {min }}$ \\
2 & $\mathcal{G}_{2}$ & $E_{8}$ & $C_{2}=(8,4,4)$ & $4 \delta_{\text {min }}$ \\
3 & $\mathcal{G}_{3}$ & $L_{8}$ & $C_{3}=(8,2,4)$ & $8 \delta_{\text {min }}$ \\
4 & $\mathcal{G}_{4}=2 \mathcal{G}$ & $2 \mathbb{Z}^{8}$ & $C_{4}=(8,0, \infty)$ & $16 \delta_{\text {min }}$ \\
\hline
\end{tabular}

TABLE I

THE GOLDEN CODE PARTITION CHAIN WITH CORRESPONDING LATTICES, BINARY CODES, AND MINIMUM SQUARED DETERMINANTS.

The coset leaders in $\left[C_{k} / C_{k+1}\right]$ form a group of order 4 $(\mathbb{Z} / 2 \mathbb{Z} \times \mathbb{Z} / 2 \mathbb{Z})$, which is generated by two binary generating vectors $\mathbf{h}_{1}$ and $\mathbf{h}_{2}$

$$
\left[C_{k} / C_{k+1}\right]=\left\{b_{1} \mathbf{h}_{1}+b_{2} \mathbf{h}_{2} \mid b_{1}, b_{2} \in G F(2)\right\}
$$

If we consider all the lattices in (10), and the corresponding nested sequence of linear binary codes $C_{k}$, we have:

$$
\begin{aligned}
& {\left[C_{0} / C_{1}\right]:\left\{\begin{array}{l}
\mathbf{h}_{1}^{(1)}=(0,0,0,0,0,0,0,1) \\
\mathbf{h}_{2}^{(1)}=(0,0,0,1,0,0,0,0)
\end{array}\right.} \\
& {\left[C_{1} / C_{2}\right]:\left\{\begin{array}{l}
\mathbf{h}_{1}^{(2)}=(0,0,0,0,0,0,1,1) \\
\mathbf{h}_{2}^{(2)}=(0,0,0,0,0,1,0,1)
\end{array}\right.} \\
& {\left[C_{2} / C_{3}\right]:\left\{\begin{array}{l}
\mathbf{h}_{1}^{(3)}=(0,1,0,1,0,1,0,1) \\
\mathbf{h}_{2}^{(3)}=(0,0,1,1,0,0,1,1)
\end{array}\right.} \\
& {\left[C_{3} / C_{4}\right]:\left\{\begin{array}{l}
\mathbf{h}_{1}^{(4)}=(0,0,0,0,1,1,1,1) \\
\mathbf{h}_{2}^{(4)}=(1,1,1,1,1,1,1,1)
\end{array}\right.}
\end{aligned}
$$

Encoder structure - Fig. 1 shows the encoder structure of the proposed concatenated scheme. The input bits feed two encoders, an upper trellis encoder and a lower lattice encoder.

For two lattices $\Lambda_{\ell_{0}}$ and $\Lambda_{\ell_{0}+\ell}$, we have the quotient group $\Lambda_{\ell_{0}} / \Lambda_{\ell_{0}+\ell}$ with order $N_{c}=\left|\Lambda_{\ell_{0}} / \Lambda_{\ell_{0}+\ell}\right|=4^{\ell}$, which corresponds to the total number of cosets of the sublattice $\Lambda_{\ell_{0}+\ell}$ in 
the lattice $\Lambda_{\ell_{0}}$. We assume that we have $4 q$ input bits. The upper encoder is a trellis encoder that operates on $q_{1}$ information bits. Given the relative partition depth $\ell$, we select a trellis code rate $R_{c}=1 / \ell$. The trellis encoder outputs $n_{c}=q_{1} / R_{c}$ bits, which are used by the coset mapper to label the coset leader $\mathbf{c}_{1} \in\left[\Lambda_{\ell_{0}} / \Lambda_{\ell_{0}+\ell}\right]$. The mapping is obtained by the product of the $n_{c}$ bit vector with a binary coset leader generator matrix $H_{1}$ with rows $\mathbf{h}_{1}^{\left(\ell_{0}+1\right)}, \mathbf{h}_{2}^{\left(\ell_{0}+1\right)}, \cdots, \mathbf{h}_{1}^{\left(\ell_{0}+\ell\right)}, \mathbf{h}_{2}^{\left(\ell_{0}+\ell\right)}$, where the rows are taken from (11). This will limit $q_{1}=2$.

The lower encoder is a sublattice encoder for $\Lambda_{\ell_{0}+\ell}$ and operates on $q_{2}+q_{3}$ information bits, where $q_{2}=2 \times$ $\left(4-\ell-\ell_{0}\right)$ and $q_{3}=4 q-q_{1}-q_{2}$. The $q_{2}$ bits label the cosets of $2 \mathbb{Z}^{8}$ in $\Lambda_{\ell_{0}+\ell}$ by multiplying the matrix $H_{2}$ with rows $\mathbf{h}_{1}^{\left(\ell_{0}+\ell+1\right)}, \mathbf{h}_{2}^{\left(\ell_{0}+\ell+1\right)}, \cdots, \mathbf{h}_{1}^{(4)}, \mathbf{h}_{2}^{(4)}$, which generates the coset leaders $\mathbf{c}_{2} \in\left[\Lambda_{\ell_{0}+\ell} / 2 \mathbb{Z}^{8}\right]$.

We finally add both coset leaders of $\mathbf{c}_{1}$ and $\mathbf{c}_{2}$ modulo 2 to get $\mathbf{c}^{\prime}$. The $q_{3}$ bits go through the $2 \mathbb{Z}^{8}$ encoder and generate vector $2 \mathbf{u}, \mathbf{u} \in \mathbb{Z}^{8}$, which is added to $\mathbf{c}^{\prime}$ (lifted to have integer components) and then mapped to the Golden codeword $X_{t}$.

We now focus on the structure of the trellis code to be used. We consider linear convolutional encoders over the quaternary alphabet $\mathbb{Z}_{4}=\{0,1,2,3\}$ with $\bmod 4$ operations, in order to match the four way partitions. We assume the natural mapping between pairs of bits and $\mathbb{Z}_{4}$ symbols, i.e., $0 \rightarrow 00,1 \rightarrow$ $01,2 \rightarrow 10,3 \rightarrow 11$. Let $\beta \in \mathbb{Z}_{4}$ denote the input symbol and $\alpha_{1}, \ldots, \alpha_{\ell} \in \mathbb{Z}_{4}$ denote the $\ell$ output symbols generated by the generator polynomials $g_{1}(D), \ldots g_{\ell}(D)$ over $\mathbb{Z}_{4}$.

Trellis labeling- In this subsection, we focus on slow block fading cases. For fast fading cases, it can be derived similarly by replacing the sums with the products in $\Delta_{\text {min }}^{\prime}$.

In order to increase the potential coding gain, the lower bound $\Delta_{\min }^{\prime}$ in (6) should be maximized. Let $\Delta_{\text {par }}=2^{\ell_{0}+\ell} \delta_{\text {min }}$ denote the minimum determinant of the trellis parallel transitions corresponding to the Golden codewords in the partition $\Lambda_{\ell_{0}+\ell}+\mathbf{c v}_{1}$. Let $\Delta_{\text {sim }}=$ $\min _{\mathbf{X} \neq \mathbf{0}_{2 \times 2 L}} \sum_{t=t_{o}}^{t_{o}+L^{\prime}-1} \operatorname{det}\left(X_{t} X_{t}^{\dagger}\right)$ denote the minimum determinant on the shortest simple error event, where $L^{\prime}$ is the length of the shortest simple error event diverging from the zero state at $t_{o}$ and merging to the zero state at $t_{i}=t_{o}+L^{\prime}-1$.

The lower bound $\Delta_{\min }^{\prime}$ in (6) is determined either by the parallel transition error events or by the shortest simple error events in the trellis, i.e.,

$$
\begin{gathered}
\Delta_{\text {min }}^{\prime}=\min \left\{\Delta_{\mathrm{par}}, \Delta_{\mathrm{sim}}\right\} \\
\geq \min \left\{\Delta_{\mathrm{par}}, \min _{X_{t_{o}}} \operatorname{det}\left(X_{t_{o}} X_{t_{o}}^{\dagger}\right)+\min _{X_{t_{i}}} \operatorname{det}\left(X_{t_{i}} X_{t_{i}}^{\dagger}\right)\right\} .
\end{gathered}
$$

For fast block fading the above sum of two terms is replaced by a product of the same two terms. The corresponding coding gain will be

$$
\gamma_{a s}^{\prime}=\min \left\{\gamma_{a s}^{\prime}\left(\Delta_{\text {par }}\right), \gamma_{a s}^{\prime}\left(\Delta_{\text {sim }}\right)\right\}
$$

Therefore, we focus on $\Delta_{\min }^{\prime}$ and we have the following:

Design Criterion - The incoming and outgoing branches for each state should belong to different cosets that have the common father node as deep as possible in the partition tree.

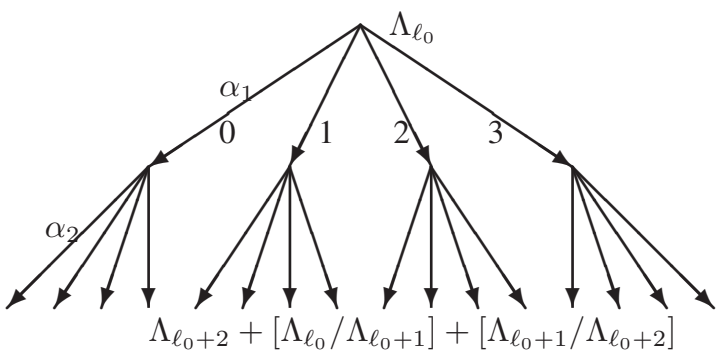

Fig. 2. Two level $(\ell=2)$ partition tree of $\Lambda_{\ell_{0}}$ into 16 cosets of $\Lambda_{\ell_{0}+2}$.

This guarantees that simple error events in the trellis give the largest contribution to $\Delta_{\min }^{\prime}$.

In order to fully satisfy the above criterion for a given relative partition level $\ell$, the minimum number of trellis states should be $N_{c}=4^{\ell}$. In order to reduce complexity we will also consider trellis codes with fewer states. We will see in the following that the performance loss of these suboptimal codes (in terms of the above design rule) is marginal since $\Delta_{\text {par }}$ is dominating the code performance. Nevertheless, the optimization of $\Delta_{\text {sim }}$ yields a performance enhancement. In fact, maximizing $\Delta_{\text {sim }}$ has the effect of minimizing another relevant term in the determinant spectrum.

Decoding - The decoder is structured as a typical TCM decoder, i.e. a Viterbi algorithm using a branch metric computer. The branch metric computer should output the distance of the received symbol from all the cosets of $\Lambda_{\ell_{0}+\ell}$ in $\Lambda_{\ell_{0}}$.

\section{CODE DESIGN EXAMPLE}

In this section, we give an example to show the performance of GST-TCM with different numbers of trellis states over slow and fast block fading channels.

We assume each frame contains $L=130$ symbols $(2 \times 2)$. We first describe the uncoded Golden code scheme with the same frame length, which is used as a reference system for performance comparison.

Uncoded Golden code 6bpcu - A total of 12 bits must be sent in a Golden codeword (3): the symbols $a, b, c, d$ are in a 8-QAM (3bits). This guarantees that the same average energy is transmitted from both antennas. In this case we have $E_{s, 2}=1.5$ and $q=3$ bits.

GST-TCM Example - Consider $\Lambda_{\ell_{0}}=\mathbb{Z}^{8}, \Lambda_{\ell_{0}+\ell}=L_{8}$, where $\ell_{0}=0, \ell=3$. Using 16-QAM we have $6 \mathrm{bpcu}, E_{s, 1}=$ 2.5 and $q=3$ bits. Since we have a three level partition with quotient group $\Lambda_{\ell} / \Lambda_{\ell_{0}+\ell}=\mathbb{Z}^{8} / L_{8}$ of order $N_{c}=64$ we use quaternary trellis encoders with rate $R_{c}=1 / 3$. Then we have $q_{1}=2$ input information bits and $n_{c}=6$ output bits, which label the 64 coset leaders. The sublattice encoder has $q_{2}=2$ and $q_{3}=8$ input bits, giving a total number of input bits per information symbol $q=\left(q_{1}+q_{2}+q_{3}\right) / 4=12 / 4=3$ bits, i.e., 6 bpcu.

The 16 state GST-TCM has the following generator polynomials: $g_{1}(D)=D, g_{2}(D)=D^{2}, g_{3}(D)=1+D^{2}$, where $D$ is a delay operator. For the 16 state GST-TCM, at each trellis state, four outgoing branches are labeled with $\alpha_{1}, \alpha_{2}, \alpha_{3}$, corresponding to input $\beta \in \mathbb{Z}_{4}$. In this case, since $\alpha_{1}$ and 


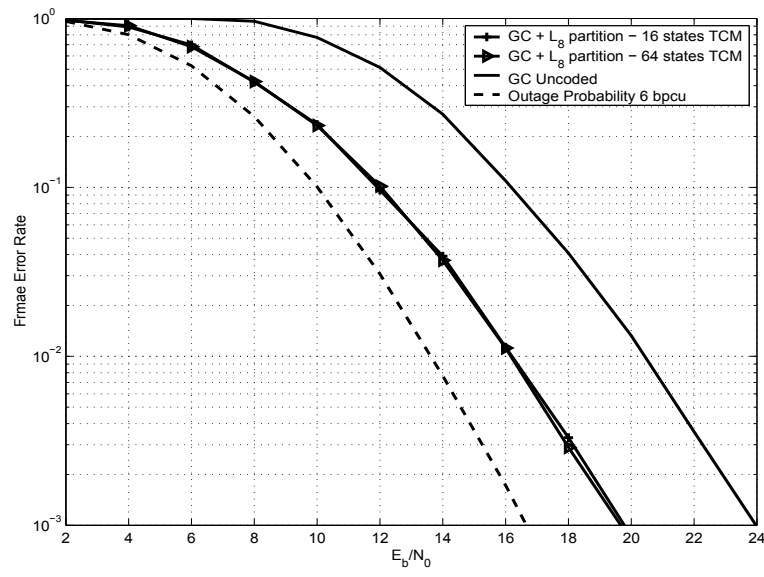

Fig. 3. Performance comparison of 16- and 64-state trellis codes using 16QAM constellation and an uncoded transmission at the rate 6bpcu, $\Lambda=\mathbb{Z}^{8}$, $\Lambda_{\ell}=L_{8}, \ell=3$, slow block fading.

$\alpha_{2}$ are fixed, $\alpha_{3}$ varies. This guarantees an increased $\Delta_{\text {min }}^{\prime}$. The four trellis branches arriving in each state are in cosets of $E_{8}$. This does not give the highest possible increase to $\Delta_{\min }^{\prime}$ since $\alpha_{2}$ varies. This results in a suboptimal design, which yields

$$
\Delta_{\min }^{\prime} \geq \min \left(8 \delta_{\min }, 4 \delta_{\min }+\delta_{\min }+2 \delta_{\min }\right)=7 \delta_{\min } .
$$

The above problem suggests the use of a 64 state encoder with the generator polynomials: $g_{1}(D)=D, g_{2}(D)=$ $D^{2}, g_{3}(D)=1+D^{3}$. In such a case, the output labels $\alpha_{1}, \alpha_{2}$ are fixed for all outgoing and incoming states. Only $\alpha_{3}$ varies to choose different subgroups from the deepest partition level in this example. This fully satisfies our design rule and yields $\Delta_{\min }^{\prime} \geq \min \left(8 \delta_{\min }, 4 \delta_{\min }+\delta_{\min }+2 \delta_{\min }+4 \delta_{\min }\right)=8 \delta_{\min }$.

Compared to 16 state GST-TCM, the 64 state GST-TCM has a higher decoding complexity. It requires $N_{c}=256$ lattice decoding operations in each trellis section, while the 16 state TCM only requires $N_{c}=64$.

Performance of the proposed codes and the uncoded scheme with 6 bpcu over a slow block fading channel is compared in Fig. 3. We can observe that a 16 state GST-TCM outperforms the uncoded scheme by $4.2 \mathrm{~dB}$ and is $3.1 \mathrm{~dB}$ away from outage probability at the FER of $10^{-3}$. The 64 state GSTTCM outperforms the uncoded case by $4.3 \mathrm{~dB}$ and $3 \mathrm{~dB}$ away from outage probability at FER of $10^{-3}$.

In the case os fast fading the same codes as above are compared in Fig. 4. It is shown that the 16 and 64 state codes have the same performance and outperform the uncoded Golden code by about $4.5 \mathrm{~dB}$ at the FER of $10^{-3}$.

\section{CONCLUSIONS}

In this paper, we presented GST-TCM, a coding scheme suitable for $2 \times 2$ MIMO systems over slow and fast block fading channels. The inner modulation is the Golden code, which provides the full diversity and non-vanishing determinant property. Lattice set partitioning is designed specifically to increase

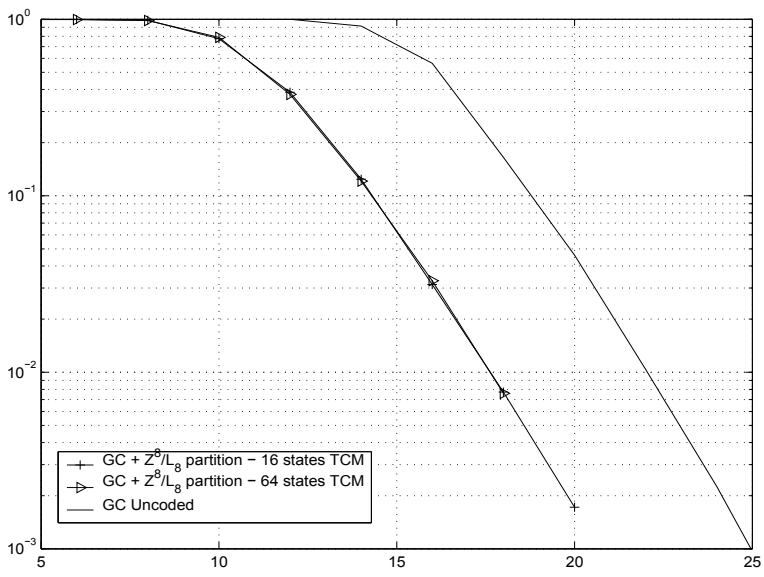

Fig. 4. Performance comparison of 16- and 64-state trellis codes using 16QAM constellation and an uncoded transmission at the rate 6 bpcu, $\Lambda=\mathbb{Z}^{8}$, $\Lambda_{\ell}=L_{8}, \ell=3$, fast block fading.

the minimum determinant of the Golden codewords, which label the branches of the trellis code. Viterbi algorithm is applied in trellis decoding, where branch metrics are computed by using a lattice decoder. The general framework for GSTTCM design and optimization is based on Ungerboeck TCM design rules. It is shown that the design criteria are robust to various channel conditions ranging from slow to fast block fading.

\section{REFERENCES}

[1] V. Tarokh, N. Seshadri and A. R. Calderbank, "Space-Time Codes for High Data Rate Wireless Communications: Performance Criterion and Code Construction," IEEE Trans. on Information Theory, vol. 44, no. 2, pp. 744-765, 1998.

[2] J.-C. Belfiore, G. Rekaya, and E. Viterbo, "The Golden Code: A $2 \times$ 2 full-rate space-time code with non-vanishing determinants," IEEE Transactions on Information Theory, vol. 51, no. 4, pp. 1432-1436, Apr. 2005.

[3] D. Champion, J.-C. Belfiore, G. Rekaya and E. Viterbo, "Partitionning the Golden Code: A framework to the design of Space-Time coded modulation," Canadian Workshop on Information Theory, Montreal, 2005.

[4] A. R. Calderbank and N. J. Sloane, "New trellis codes based on lattices and cosets," IEEE Transactions on Information Theory, vol. 33, no. 2, pp. 177-195, Mar. 1987

[5] G. Ungerboeck, "Trellis Coded Modulation with Redundant Signal Sets. Part II: State of the Art," IEEE Communications Magazine, vol. 25, n. 2, pp. 12-21, Feb. 1987.

[6] G. D. Forney Jr., "Coset codes. I. Introduction and geometrical classification” IEEE Transactions on Information Theory, vol. 34, Sept. 1988, pp. 1123-1151.

[7] G. D. Forney Jr., "Coset codes. II. Binary lattices and related codes," IEEE Transactions on Information Theory, vol. 34, Sept. 1988, pp. 1152-1187.

[8] E. Biglieri, Coding for wireless channels, Springer, New York, 2005.

[9] J. H. Conway and N. J. A. Sloane, "Sphere Packings, Lattices and Groups," Springer-Verlag, New York, 1992.

[10] H. Lütkepolhl , Handbook of Matrices, Chichester, England, John Wiley \& Sons Ltd., 1996.

[11] Y. Hong, E. Viterbo, J.-C. Belfiore, "Golden Space-Time Trellis Coded Modulation", submitted to IEEE Trans. Information Theory, April 2006 (available on arXiv:0604063). 